\title{
Expected number of supergiant fast X-ray transients in the Milky Way
}

\author{
L. Ducci ${ }^{1,2}$, V. Doroshenko ${ }^{1}$, P. Romano ${ }^{3}$, A. Santangelo ${ }^{1}$, and M. Sasaki ${ }^{1}$ \\ ${ }^{1}$ Institut für Astronomie und Astrophysik, Eberhard Karls Universität, Sand 1, 72076 Tübingen, Germany \\ e-mail: ducci@astro.uni-tuebingen.de \\ 2 ISDC Data Center for Astrophysics, Université de Genève, 16 chemin d'Écogia, 1290 Versoix, Switzerland \\ 3 INAF, Istituto di Astrofisica Spaziale e Fisica Cosmica - Palermo, via U. La Malfa 153, 90146 Palermo, Italy
}

Received 15 May 2014 / Accepted 15 July 2014

\begin{abstract}
In the past fifteen years a new generation of X-ray satellites led to the discovery of a subclass of high-mass X-ray binaries (HMXBs) with supergiant companions and a peculiar transient behaviour: supergiant fast X-ray transients (SFXTs). We calculate the expected number of Galactic SFXTs for the first time, using two different statistical approaches and two sets of data based on Swift and INTEGRAL surveys, with the aim to determine how common the SFXT phenomenon really is. We find that the expected number of SFXTs in the Galaxy is $\approx 37_{-22}^{+53}$, which shows that SFXTs constitute a large portion of X-ray binaries with supergiant companions in the Galaxy. We compare our estimate with the expected number of Galactic HMXBs predicted from observations and evolutionary models and discuss the implications for the nature of SFXTs.
\end{abstract}

Key words. X-rays: binaries

\section{Introduction}

High-mass X-ray binaries (HMXBs) are a class of binary systems composed of a normal star of spectral type $\mathrm{O}$ or $\mathrm{B}$ and a degenerate star (either a neutron star or a black hole). The strong X-ray emission is caused by the accretion of a fraction of the stellar wind ejected by the donor star or through mass transfer via Roche-lobe overflow (see e.g. Treves et al. 1988, and references therein). There are about 250 known HMXBs in our Galaxy and in the Magellanic Clouds (Liu et al. 2005; Liu et al. 2006; Krivonos et al. 2012), observed over a wide range of luminosities, from $\sim 10^{32} \mathrm{erg} \mathrm{s}^{-1}$ to $\sim 10^{39} \mathrm{erg} \mathrm{s}^{-1}$. The members of this class are historically divided into two sub-classes: HMXBs with OB supergiants (hereafter supergiant X-ray binaries, SGXBs) and HMXBs with a rapidly rotating OBe mainsequence or giant star accompanied by a transient circumstellar disc.

Since the discovery of XTE J1739-302 in 1998 (Smith et al. 1998; see also Sunyaev et al. 2003) and the identification of its optical counterpart (Smith et al. 2003), a new generation of satellites observing the X-ray sky led to the discovery of SGXBs with peculiar properties, called supergiant fast X-ray transients (SFXTs; Smith et al. 2004; Sguera et al. 2005; Negueruela et al. 2006), which are the subject of this paper. In contrast to the classical SGXBs, which are mostly bright persistent sources showing moderate variability, SFXTs show bright $\left(\sim 10^{36}-10^{37} \mathrm{erg} \mathrm{s}^{-1}\right)$ and short flares (lasting from minutes to hours). The dynamic range of the flux change is three to five orders of magnitude, from a quiescent luminosity of $\sim 10^{32}-10^{33} \mathrm{erg} \mathrm{s}^{-1}$ up to the outburst peak luminosity (in't Zand 2005; Walter \& Zurita Heras 2007). The Swift monitoring of a sample of five SFXTs (Romano et al. 2007; Sidoli et al. 2008; Romano et al. 2008) showed that the short flares are the brightest part of a much longer outburst event that typically lasts a few days. In periods without outbursts, SFXTs show a fainter flaring activity with luminosities of $\sim 10^{33}-10^{34} \mathrm{erg} \mathrm{s}^{-1}$. The X-ray spectra during outbursts can be fitted with models that are commonly used to describe the X-ray emission from HMXBs hosting neutron stars, such as an absorbed power-law with cut-offs at $\sim 15-30 \mathrm{keV}$ (e.g. Walter \& Zurita Heras 2007).

Although many SFXTs are in general remarkably different from the classical SGXBs, some of them, the so-called intermediate SFXTs, have properties similar to some SGXBs, such as Vela X-1 (Kreykenbohm et al. 2008; Doroshenko et al. 2011), and 4U 1907-09 (Doroshenko et al. 2012). Doroshenko et al. $(2011,2012)$ attempted to link the dipping activity observed in Vela X-1 and 4U 1907-09 to the flaring activity of SFXTs. They suggested that these two sources might constitute a missing link between the SGXBs and SFXTs.

Liu et al. (2011) proposed that some of the SFXTs might have evolved from Be/X-ray binaries. However, it is still not clear if SFXTs come from an evolutionary path that is different from that of classical SGXBs.

Several accretion models have been proposed to explain the properties of SFXTs. Nonetheless, a decisive explanation of the peculiar transient behaviour of these sources is still lacking. The proposed mechanisms are related to the properties of either the supergiant wind or the accreting neutron star. A first set of models involves accretion from a spherically symmetric inhomogeneous or anisotropic wind, where the flares are produced by the accretion of denser regions of matter expelled from the donor star (e.g. in't Zand 2005; Negueruela et al. 2008; Walter \& Zurita Heras 2007; Sidoli et al. 2007; Chaty 2013). The second set of models involves accretion-gating mechanisms, where the direct accretion onto a neutron star is halted by a magnetic or centrifugal barrier (depending on the spin period and the magnetic field at the surface of the neutron star) and the high dynamic range is caused by transitions through different regimes of 
accretion (Grebenev \& Sunyaev 2007; Bozzo et al. 2008; Ducci et al. 2010; Lutovinov et al. 2013; Drave et al. 2014, and references therein). Other proposed scenarios associate the flaring activity with the formation and dissipation of temporary accretion discs (Smith et al. 2006; Ducci et al. 2010, and references therein).

Several studies on the expected number of Galactic HMXBs and their X-ray luminosity functions have been made. These works tackle the problem either from observational (e.g. Voss \& Ajello 2010; Lutovinov et al. 2013; Doroshenko et al. 2014) or evolutionary points of view (e.g. Lipunov et al. 2009; Belczynski et al. 2008; Iben et al. 1995; Dalton \& Sarazin 1995; van den Heuvel 1976; Meurs \& van den Heuvel 1989). Except for the study of van den Heuvel (2012), who concluded that the number of discovered SFXTs supports the number of HMXBs obtained from evolutionary models, the expected number of SFXTs in the Galaxy has not been determined yet, nor have they been compared with the results of these previous works.

The aim of this paper is to evaluate the expected number of SFXTs in the Galaxy to understand whether the SFXTs are only a small sample of a very special case or rather a significant subclass of HMXBs. The methods and input information adopted and the results are shown in Sect. 2. In Sect. 3 the results and a comparison between the expected number of Galactic SFXTs calculated in this work and the expected number of Galactic HMXBs derived in other studies are discussed.

\section{Calculations}

We calculated the total number of SFXTs in the Galaxy adopting two different approaches:

- a semi-analytical method, where the outbursts of SFXTs are treated as events occurring in a given interval of time with a known average rate that is independent of the time of the observation;

- a series of Monte Carlo simulations of a population of HMXBs with the flaring properties of known SFXTs. This part of the work is based on the novel method of modelling XRB populations of Doroshenko et al. (2014).

For the calculation we relied on two datasets: the 100-month Swift/BAT catalogue (Romano et al. 2014, hereafter R14; see Sect. 2.1) and the cumulative luminosity distributions and duty cycles of SFXTs obtained by Paizis \& Sidoli (2014, hereafter P14) from a systematic analysis of about nine years of INTEGRAL data (17-100 keV).

The two datasets are based on all-sky hard X-ray surveys. The Burst Alert Telescope (BAT; Barthelmy et al. 2005) on board the NASA satellite Swift (Gehrels et al. 2004) is a coded mask telescope with a field of view of $\sim 2.29$ sr down to $5 \%$ coding fraction (percentage of the detector array shadowed by the mask). It operates over the 15-150 keV energy band (Krimm et al. 2013). The Imager on Board INTEGRAL Satellite (IBIS; Ubertini et al. 2003) is one of the telescopes on board the ESA satellite INTErnational Gamma-Ray Astrophysics Laboratory (INTEGRAL; Winkler et al. 2003). It is a coded mask telescope with a field of view of $29^{\circ} \times 29^{\circ}$ and is composed of two detectors: the INTEGRAL Soft Gamma-Ray Imager (ISGRI; Lebrun et al. 2003) which operates in 15-400 keV band, and the Pixellated Imaging Coesium Iodide Telescope (PICsIT; Di Cocco et al. 2003) which operates in the 180-2000 keV band.

The wide fields of view and high sensitivities of these two instruments are very useful for serendipitous surveys. Therefore, they frequently observed SFXTs in outburst. In particular, IBIS/ISGRI has a better short-term ( $\sim 2 \mathrm{ks})$ sensitivity than BAT. On the other hand, BAT provides a more uniform exposure over the Galactic plane thanks to the wider field of view.

Despite the favourable characteristics provided by these and other X-ray satellites for this type of studies, the detection of the entire population is difficult because of the peculiar transient properties of SFXTs. Therefore, a part of the population of Galactic SFXTs (especially those with lower outburst rates and larger distances) has most likely not been detected yet.

\subsection{Swift/BAT}

The total number of SFXTs in the Galaxy can be derived statistically from the number of the observed SFXTs and their outburst rates. Our calculation is based on the method developed by Muno et al. (2008) to constrain the number of Galactic magnetars. As input data we used the detections in excess of $5 \sigma$ (corresponding to a $15-50 \mathrm{keV}$ limiting flux of $F_{\min } \approx 8.24 \times$ $10^{-10} \mathrm{erg} \mathrm{cm}^{-2} \mathrm{~s}^{-1}$ ) from R14. An outburst event produced by a SFXT typically contains several flares that can lead to several detections per day. Since our calculation requires the number of observed outbursts for any given SFXT, if more than one detection occurred in a day we considered only the detection with the highest flux. In Table 1 the number of BAT orbital-averaged detections of outbursts $N_{\mathrm{BAT}}$ and the outburst rate $r=N_{\mathrm{BAT}} / T$, where $T=287.7 \mathrm{~d}$ is the average monitoring period, are reported for all the SFXTs detected with BAT.

The cumulative luminosity distribution of the observed flares of SFXTs varies from source to source (see P14 and R14). For each SFXT, the lowest detected luminosity depends on the sensitivity of the instrument and the distance of the source from the observer. Therefore, relatively distant SFXTs with intrinsically low outburst rate have most likely not been detected at all. An example is IGR J11215-5952, a peculiar SFXT at $\approx 7.3 \mathrm{kpc}$ (Coleiro \& Chaty 2013) which shows periodic bright flares $\left(P_{\text {orb }} \approx 165 \mathrm{~d}\right.$; outburst rate $r=0.006 \mathrm{~d}^{-1}$; Romano et al. 2009). It was never detected by BAT because of its relatively low $15-50 \mathrm{keV}$ flux during the outbursts $(\approx 2-12 \times$ $10^{-10}$ erg $\mathrm{cm}^{-2} \mathrm{~s}^{-1}$; Lubiński et al. 2005; Sidoli et al. 2006). The first step of our calculation was therefore to estimate the fraction of the Galaxy completely surveyed by BAT for SFXTs that have properties of the observed SFXT sample. The prototypical $^{1}$ SFXT SAX J1818.6-1703 shows the lowest luminosities among all the SFXTs detected by BAT. Therefore, we calculated the largest distance that an SFXT with the same properties as SAX J1818.6-1703 can have to still be detected by BAT with a confidence level of $90 \%$. According to Poisson statistics, the probability that any given SFXT is detected is $p=1-\mathrm{e}^{-N}$. Assuming $p=0.9$, we find $N \approx 2.3$. Since $N$ is a discrete variable, for the following calculation of $f$ we approximated $N$ as $N \sim 2$. From the BAT luminosity distribution of SAX J1818.6-1703 we find that the luminosities corresponding to the two detections with highest fluxes are higher than $L_{\text {min }} \approx 1.4 \times 10^{36} \mathrm{erg} \mathrm{s}^{-1}(15-50 \mathrm{keV})$. Flares with these luminosities can be observed by BAT up to a distance of $R_{\text {loc }}=\sqrt{L_{\min } /\left(4 \pi F_{\min }\right)} \approx 3.8 \mathrm{kpc}$. Using the model for stellar mass distribution of the Galaxy of Cordes \& Lazio (2002), $R_{\text {loc }}$ derived above, and the BAT sky coverage of 80-94\% day ${ }^{-1}$ (Krimm et al. 2013), we found that the fraction of stellar mass

1 Prototypical SFXTs have a dynamic range exceeding $10^{3}$ (see e.g. P14). In addition to SAX J1818.6-1703 the other prototypical SFXTs are XTE J1739-302, IGR J17544-2619, and IGR J08408-4503. 
Table 1. Orbitally averaged detections $(>5 \sigma)$ throughout the Swift mission (2005-02-12 to 2013-05-31) from R14.

\begin{tabular}{|c|c|c|c|c|}
\hline \multirow[t]{2}{*}{ Name } & \multicolumn{2}{|c|}{ Distance } & \multirow{2}{*}{$\begin{array}{c}N_{\text {BAT }} \\
>5 \sigma \\
\text { orbital }\end{array}$} & \multirow{2}{*}{$\begin{array}{c}r \\
\left(\mathrm{~d}^{-1}\right)\end{array}$} \\
\hline & $(\mathrm{kpc})$ & Ref. & & \\
\hline IGR J08408-4503 & $3.4 \pm 0.3$ & (1) & 5 & 0.017 \\
\hline IGR J16328-4726 & 3-10 & (2) & 3 & 0.010 \\
\hline IGR J16418-4532 & 13 & (3) & 16 & 0.054 \\
\hline IGR J16465-4507 & $12.7 \pm 1.3$ & (1) & 1 & 0.003 \\
\hline IGR J16479-4514 & 4.9 & (4) & 61 & 0.208 \\
\hline XTE J1739-302 & 2.7 & (4) & 29 & 0.102 \\
\hline IGR J17544-2619 & 3.6 & (4) & 23 & 0.083 \\
\hline SAX J1818.6-1703 & 2.1 & (5) & 17 & 0.062 \\
\hline AX J1841.0-0536 & $7.8 \pm 0.7$ & (1) & 16 & 0.055 \\
\hline AX J1845.0-0433 & 3.6 & (6) & 8 & 0.028 \\
\hline IGR J18483-0311 & 2.8 & (5) & 24 & 0.083 \\
\hline
\end{tabular}

Notes. When more than one detection was made on a given day, only one detection has been counted.

References. (1) Coleiro \& Chaty (2013); (2) Fiocchi et al. (2013); (3) Chaty et al. (2008); (4) Rahoui et al. (2008); (5) Torrejón et al. (2010); (6) Coe et al. (1996).

in the spiral arms surveyed by BAT for SFXTs each day is $f=11-13 \%$. Taking into account the position of the SFXTs detected by BAT in the Galactic plane, $s=5 \pm 1$ of them are surveyed each day by BAT.

The probability that an SFXT in the region of the Galaxy surveyed by BAT is detected during an outburst is $1-\mathrm{e}^{-r T}$, where $r$ is the outburst rate reported in Table 1. According to the binomial distribution, given $n \geq s$ SFXTs in the surveyed region and $N \geq n$ SFXTs in the Galaxy, the probability that $s$ SFXTs are detected in outburst is:

$p(s \mid r T, f, N)=\sum_{n=s}^{N}\left[\left(1-\mathrm{e}^{-r T}\right)^{s}\left(\mathrm{e}^{-r T}\right)^{n-s}\left(\begin{array}{l}n \\ s\end{array}\right) \times f^{n}(1-f)^{N-n}\left(\begin{array}{l}N \\ n\end{array}\right)\right]$

where the first part of the right-hand side of Eq. (1) is the probability that $s$ out of $n$ SFXTs in the surveyed region are detected and the second part is the probability that $n$ out of $N$ SFXTs are located in a fraction $f$ of the Galaxy.

According to the Bayes theorem, the conditional probability $p(s \mid r T, f, N)$ defined in Eq. (1) can be inverted to obtain $p(N \mid r T, f, s)$, namely the probability that there are $N$ SFXTs given that $s$ are found in a fraction $f$ of the Galaxy. In our calculations, we assumed a uniform prior distribution (for a similar application of this method, on magnetars, see Muno et al. 2008).

Given the average outburst rate $\bar{r}=0.06 \mathrm{~d}^{-1}$ (see Table 1) of the SFXTs within $R_{\mathrm{loc}}$ and assuming $s=5 \pm 1, T=287.7 \mathrm{~d}$, and $f$ ranging from $11 \%$ to $13 \%$, we obtained that the total number of SFXTs in the Galaxy for the given average outburst rate is $N_{\overline{\mathrm{r}}}=37_{-22}^{+53}$ (uncertainties at $90 \%$ confidence level).

The SFXTs used for this calculation have outburst rates ranging from $\approx 0.017 \mathrm{~d}^{-1}$ to $\approx 0.2 \mathrm{~d}^{-1}$. However, we know that there are other SFXTs and candidate SFXTs with lower outburst rates. In addition to IGR J11215-5952 with $r=0.006 \mathrm{~d}^{-1}$, INTEGRAL detected other SFXTs candidates: IGR J20188+ 3647, IGR J18159-3353, IGR J21117+3427 (Sguera et al. 2006 and Bodaghee et al. 2007), IGR J17541-2252 (Turler et al. 2006 and Bodaghee et al. 2007), IGR J10043-8702, IGR J105006410, IGR J15283-4443 (Paizis et al. 2006), IGR J18462-0223

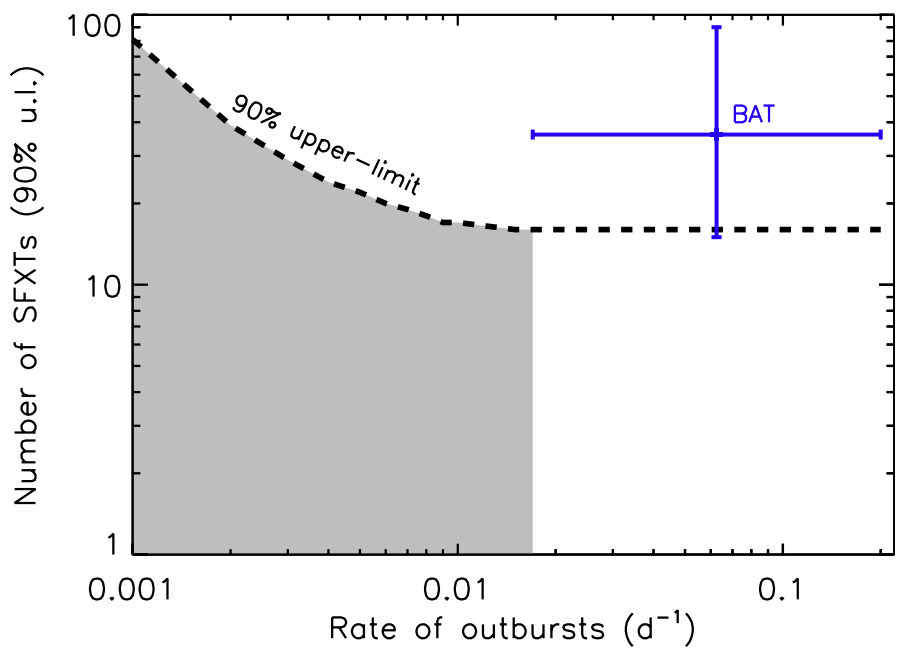

Fig. 1. 90\% upper limits of the total number of SFXTs in the Galaxy as a function of the outburst rate. The dashed line corresponds to the $90 \%$ upper limits of $N$ obtained from the probability distribution $p(N \mid r T, f, s)$. The grey area shows for each assumed outburst rate, the allowed $N$, given $s=0, T=287.7 \mathrm{~d}$, and $f$ ranging from $11 \%$ to $13 \%$. The value of $N_{\overline{\mathrm{r}}}$ obtained assuming $\bar{r}=0.06 \mathrm{~d}^{-1}, s=5 \pm 1$, $T=287.7 \mathrm{~d}$, and $f=11-13 \%$ is also plotted.

(Grebenev et al. 2007). They have been detected only once in $~ 11$ years (except for IGR J18462-0223), therefore their outburst rates are likely lower than $0.017 \mathrm{~d}^{-1}$. Driven by the high number of SFXTs and candidate SFXTs with $r \lesssim 0.02 \mathrm{~d}^{-1}$, we calculated the $90 \%$ complete upper limits of the number of Galactic SFXTs with outburst rates in the range $0.001 \leq$ $r \leq 0.017 \mathrm{~d}^{-1}$. In Fig. 1 we plot the upper-limits of the expected number of Galactic SFXTs as a function of the outburst rate (dashed line). We derived the upper limits from the probability distribution $p(N \mid r T, f, s)$ that can be obtained by inverting Eq. (1) and assuming $s=0$ (because BAT did not detect any SFXT with $r<0.017 \mathrm{~d}^{-1}$ within $\left.R_{\text {loc }}\right), T=287.7 \mathrm{~d}$, and $f=11-13 \%$. The grey region in Fig. 1 shows the solutions allowed by the probability distribution $p(N \mid r T, f, s)$ for $s=0$ and $0.001 \leq r \leq 0.017 \mathrm{~d}^{-1}$. The value of $N_{\overline{\mathrm{r}}}$ obtained above is also plotted.

\subsection{INTEGRAL}

A large portion of all observed SFXTs has been discovered by INTEGRAL. The reason is that while the cumulative sensitivies of Swift/BAT and INTEGRAL/ISGRI surveys are similar, the latter has a sensitivity that is a factor of four better on the short time-scales relevant for detecting SFXT flares. Therefore, we also estimated the number of the missing SFXTs using the INTEGRAL data. Unfortunately, the INTEGRAL/ISGRI exposure is distributed much less uniformly across the sky than for Swift/BAT, which has to be taken into account. This is not trivial with the simple approach presented above, therefore we used a series of Monte Carlo simulations instead. This also allowed us to independently verify the results obtained above with a different method.

We assumed that SFXTs have the same spatial distribution as classical HMXBs (Grimm et al. 2002; Coleiro \& Chaty 2013; Doroshenko et al. 2014) and the same flaring properties as the sample of known SFXTs, which have been investigated recently by $\mathrm{P} 14$. Based on the assumed spatial distribution, we assigned a random position within the Milky Way for each simulated 
source and calculated the survey exposure in the respective direction. We assumed that SFXTs can only be detected in flaring state, and the effective flare exposure was calculated by multiplying the total exposure with the duty cycle randomly chosen among the values derived for known SFXTs (see Table 3 of P14). P14 assumed an average flare duration corresponding to a science window ( $\mathrm{ScW}$ ) length, hence the number of flares expected to occur during the survey from a given SFXT equals the number of ScWs of Table 2 of P14. For each flare we assigned a luminosity randomly sampled from one of the cumulative flare luminosity distribution functions reported by P14 (randomly chosen for each source). Distances to the simulated sources are known, which makes it trivial to calculate the flare flux distribution and highest flux for each source. The sensitivity of ISGRI on an ScW timescale is $\sim 2 \times 10^{-10} \mathrm{erg} \mathrm{cm}^{-2} \mathrm{~s}^{-1}(17-60 \mathrm{keV})$, therefore we assume that any flares with a flux exceeding this limit will be detected. As an estimate of the total ISGRI exposure we took the exposure maps from the $\sim$ nine-year Galactic plane survey (Krivonos et al. 2012).

We applied a similar approach to Swift/BAT, where we assumed slightly lower sensitivity to flares of $8 \times$ $10^{-10} \mathrm{erg} \mathrm{cm}^{-2} \mathrm{~s}^{-1}$, and a uniform exposure of $287.7 \mathrm{~d}$. The main difference with the simpler estimation presented above is that we imposed no restriction on distance to the known sources.

We found that both INTEGRAL and Swift are expected to detect at least once $46(2) \%$ of the SFXTs in Galaxy. Of the known confirmed SFXTs, IGR J08408-4503 has the lowest number of ScWs, three, where the source is detected by INTEGRAL. If we take three as the minimum number of detections required to consider a source as an SFXT (i.e. the brightest flares must be detected), INTEGRAL is expected to detect $\sim 27 \%$ of SFXTs in the Galaxy. There are 14 confirmed SFXTs ${ }^{2}$ and eight candidates (seven of which are observed only once), which makes the total expected number of SFXTs in the Milky Way between 29 and 52 .

\section{Discussion}

We applied two methods to Swift/BAT and INTEGRAL/ISGRI data to derive the total number of Galactic SFXTs. The numbers obtained with these methods agree. We found that the total number of SFXTs in the Galaxy is $\approx 37_{-22}^{+53}$. We note that this estimate strongly depends on the luminosity distribution of the flares, which is reasonably well constrained, however. Since INTEGRAL is more likely to detect brighter flares and the portion of sources detectable with INTEGRAL and Swift decreases with average flare luminosity, there may be more SFXTs in Galaxy. On the other hand, there are probably fewer than three hundred HMXBs in the Milky Way (Doroshenko et al. 2014), which makes our result of 15-90 SFXTs seem reasonable.

The fraction of SFXTs in SGXBs is fundamentally related to their evolutionary origin, therefore it would be interesting to estimate it observationally and compare it with theoretical predictions for various models. As mentioned in Sect. 1, the peculiar properties of SFXTs are commonly associated either with wind properties or with inhibition of the accretion by the neutron star centrifugal and magnetic barriers (Bozzo et al. 2008; see also Chaty 2013, for a review). In the latter case, it is easy to estimate the expected fraction of SFXTs among the SGXBs. Meurs \& van den Heuvel (1989), Dalton \& Sarazin (1995), and Iben et al. (1995) calculated the expected

\footnotetext{
2 In addition to the SFXTs listed in Table 1, the other three SFXTs are IGR J11215-5952, IGR J16195-4945, and IGR J17354-3255.
}

number of massive X-ray binaries in the Galaxy. In particular, Dalton \& Sarazin (1995) modelled the HMXB population in the Galaxy for different evolutionary scenarios (initial mass function slope $\Gamma$, birthrate, binary fraction, distribution of binary separations and binary mass ratios, and the fraction $f_{\dot{M}}$ of the matter lost from a binary system). The number of HMXBs with $L_{\mathrm{x}} \gtrsim 10^{35} \mathrm{erg} \mathrm{s}^{-1}$ predicted by Dalton \& Sarazin (1995) ranges between about 200 and 400 . In the framework of the results obtained by Lutovinov et al. (2013), who reported that the transient behaviour of SFXTs is caused by an inhibition of accretion onto the neutron star, it follows that all the observed SFXTs would be persistent with a luminosity $L_{\mathrm{x}} \gtrsim 10^{35} \mathrm{erg} \mathrm{s}^{-1}$ rather than highly variable with an observed average luminosity $\ll 10^{35} \mathrm{erg} \mathrm{s}^{-1}$. It is therefore of particular interest to compare the number of observed and predicted SFXTs + SGXBs with the estimate provided by the evolutionary model of Dalton \& Sarazin (1995) in the framework of the hypothesis of Lutovinov et al. (2013). There are 14 confirmed and 8 candidate observed SFXTs and 18 SGXBs observed with $L_{\mathrm{x}} \gtrsim 10^{35} \mathrm{erg} \mathrm{s}^{-1}$ (Lutovinov et al. 2013). From the $X$-ray luminosity function of Doroshenko et al. (2014, see Fig. 2, right panel) there are 80-100 HMXBs with $L_{\mathrm{x}} \gtrsim 10^{35} \mathrm{erg} \mathrm{s}^{-1}$. We point out that this number does not take into account SFXTs. Indeed, they have observed average luminosities $\ll 10^{35} \mathrm{erg} \mathrm{s}^{-1}$. Therefore, there are $\sim 32$ (40 including candidate SFXTs) observed SFXTs+SGXBs in total and $\sim 100-200$ predicted HMXBs (80-100 HMXBs with $L_{\mathrm{X}} \gtrsim 10^{35} \mathrm{erg} \mathrm{s}^{-1}$ plus $15-90$ SFXTs), which are consistent with the evolutionary constraints obtained by Dalton \& Sarazin (1995) for $L_{\mathrm{x}} \gtrsim 10^{35} \mathrm{erg} \mathrm{s}^{-1}$.

\section{Conclusions}

We derived for the first time the expected number of SFXTs in Galaxy. We applied two different approaches and considered two sets of data, the 100 month Swift/BAT catalogue (R14) and the results presented by $\mathrm{P} 14$ who performed a systematic analysis of $\sim$ nine-years of INTEGRAL data. The total number of SFXTs in the Galaxy is $\approx 37_{-22}^{+53}$. This value agrees with the total number of Galactic HMXBs with $L_{\mathrm{x}} \geq 10^{33} \mathrm{erg} \mathrm{s}^{-1}$ (Doroshenko et al. 2014) and with the number of expected HMXBs in the Galaxy obtained from studies of the evolution of high-mass binary systems, and shows that SFXTs represent a significant fraction of all HMXBs. In the framework of the scenario proposed by Lutovinov et al. (2013), the number of Galactic SFXTs derived in this work agrees with the expected number of HMXBs with $L_{\mathrm{x}} \geq 10^{35} \mathrm{erg} \mathrm{s}^{-1}$ obtained by Dalton \& Sarazin (1995) from evolutionary arguments (which does not take into account the effects of gating mechanisms on the observed X-ray luminosity).

Observing SFXTs in nearby galaxies is greatly complicated by their very fast transient behaviour, which makes them difficult to detect in outburst with the typical observation lengths of Chandra and XMM-Newton. Indeed, the first and only extragalactic SFXTs candidate has been only recently claimed to be observed by Laycock et al. (2014). The detection of SFXTs and an estimate of their number in the Magellanic Clouds would be important for understanding their dependence on the metallicity of a galaxy. Indeed, metallicity plays an important role in the radiatively driven wind mechanisms of massive stars and consequently in the values of mass-loss rate and velocity of the winds of donor stars of HMXBs (Lamers \& Cassinelli 1999). Since the Large and Small Magellanic Clouds have a metallicity of about $40 \%$ and $10 \%$ of that of the Milky Way, respectively, they could represent an ideal target for this type of studies. 
Acknowledgements. We thank the referee Sylvain Chaty for his useful comments, which helped to improve the paper. This work is partially supported by the Bundesministerium für Wirtschaft und Technologie through the Deutsches Zentrum für Luft und Raumfahrt (grant FKZ 50 OG 1301). V.D. and A.S. thank the Deutsches Zentrum für Luft- und Raumfahrt (DLR) and Deutsche Forschungsgemeinschaft (DFG) for financial support (grant DLR 50 OR 0702). M.S. acknowledges support by the Deutsche Forschungsgemeinschaft through the Emmy Noether Research Grant SA 2131/1-1. P.R. acknowledges contract ASI-INAF I/004/11/0. We thank H. A. Krimm and C. B. Markwardt for helpful discussions. This research has made use of the IGR Sources page maintained by J. Rodriguez \& A. Bodaghee (http://irfu.cea.fr/Sap/ IGR-Sources/).

\section{References}

Barthelmy, S. D., Barbier, L. M., Cummings, J. R., et al. 2005, Space Sci. Rev., 120, 143

Belczynski, K., Kalogera, V., Rasio, F. A., et al. 2008, ApJS, 174, 223

Bodaghee, A., Courvoisier, T. J.-L., Rodriguez, J., et al. 2007, A\&A, 467, 585

Bozzo, E., Falanga, M., \& Stella, L. 2008, ApJ, 683, 1031

Chaty, S. 2013, Adv. Space Res., 52, 2132

Chaty, S., Rahoui, F., Foellmi, C., et al. 2008, A\&A, 484, 783

Coe, M. J., Fabregat, J., Negueruela, I., Roche, P., \& Steele, I. A. 1996, MNRAS, 281,333

Coleiro, A., \& Chaty, S. 2013, ApJ, 764, 185

Cordes, J. M., \& Lazio, T. J. W. 2002 [arXiv: astro-ph/0207156]

Dalton, W. W., \& Sarazin, C. L. 1995, ApJ, 440, 280

Di Cocco, G., Caroli, E., Celesti, E., et al. 2003, A\&A, 411, L189

Doroshenko, V., Santangelo, A., \& Suleimanov, V. 2011, A\&A, 529, A52

Doroshenko, V., Santangelo, A., Ducci, L., \& Klochkov, D. 2012, A\&A, 548, A19

Doroshenko, V., Ducci, L., Santangelo, A., \& Sasaki, M. 2014, A\&A, 567, A7

Drave, S. P., Bird, A. J., Sidoli, L., et al. 2014, MNRAS, 439, 2175

Ducci, L., Sidoli, L., \& Paizis, A. 2010, MNRAS, 408, 1540

Fiocchi, M., Bazzano, A., Bird, A. J., et al. 2013, ApJ, 762, 19

Gehrels, N., Chincarini, G., Giommi, P., et al. 2004, ApJ, 611, 1005

Grebenev, S. A., \& Sunyaev, R. A. 2007, Astron Lett., 33, 149

Grebenev, S. A., Revnivtsev, M. G., \& Sunyaev, R. A. 2007, ATel, 1319, 1

Grimm, H.-J., Gilfanov, M., \& Sunyaev, R. 2002, A\&A, 391, 923

Iben, Jr., I., Tutukov, A. V., \& Yungelson, L. R. 1995, ApJS, 100, 217

in't Zand, J. J. M. 2005, A\&A, 441, L1

Kreykenbohm, I., Wilms, J., Kretschmar, P., et al. 2008, A\&A, 492, 511

Krimm, H. A., Holland, S. T., Corbet, R. H. D., et al. 2013, ApJS, 209, 14

Krivonos, R., Tsygankov, S., Lutovinov, A., et al. 2012, A\&A, 545, A27

Lamers, H. J. G. L. M., \& Cassinelli, J. P. 1999, Introduction to Stellar Winds (Cambridge: Cambridge University Press)

Laycock, S., Cappallo, R., Oram, K., \& Balchunas, A. 2014, ApJ, 789, 64

Lebrun, F., Leray, J. P., Lavocat, P., et al. 2003, A\&A, 411, L141

Lipunov, V. M., Postnov, K. A., Prokhorov, M. E., \& Bogomazov, A. I. 2009, Astron. Rep., 53, 915
Liu, Q. Z., van Paradijs, J., \& van den Heuvel, E. P. J. 2005, A\&A, 442, 1135 Liu, Q. Z., van Paradijs, J., \& van den Heuvel, E. P. J. 2006, A\&A, 455, 1165 Liu, Q. Z., Chaty, S., \& Yan, J. Z. 2011, MNRAS, 415, 3349

Lubiński, P., Cadolle Bel, M., von Kienlin, A., et al. 2005, ATel, 469, 1

Lutovinov, A. A., Revnivtsev, M. G., Tsygankov, S. S., \& Krivonos, R. A. 2013, MNRAS, 431, 327

Meurs, E. J. A., \& van den Heuvel, E. P. J. 1989, A\&A, 226, 88

Muno, M. P., Gaensler, B. M., Nechita, A., Miller, J. M., \& Slane, P. O. 2008, ApJ, 680, 639

Negueruela, I., Smith, D. M., Reig, P., Chaty, S., \& Torrejón, J. M. 2006, in The X-ray Universe 2005, ed. A. Wilson, ESA SP, 604, 165

Negueruela, I., Torrejón, J. M., Reig, P., Ribó, M., \& Smith, D. M. 2008, in A Population Explosion: The Nature \& Evolution of X-ray Binaries in Diverse Environments, eds. R. M. Bandyopadhyay, S. Wachter, D. Gelino, \& C. R. Gelino, AIP Conf. Ser., 1010, 256

Paizis, A., \& Sidoli, L. 2014, MNRAS, 439, 3439

Paizis, A., Gotz, D., Sidoli, L., Vercellone, S., \& Mereghetti, S. 2006, ATel, 865, 1

Rahoui, F., Chaty, S., Lagage, P.-O., \& Pantin, E. 2008, A\&A, 484, 801

Romano, P., Sidoli, L., Mangano, V., Mereghetti, S., \& Cusumano, G. 2007, A\&A, 469, L5

Romano, P., Sidoli, L., Mangano, V., et al. 2008, ApJ, 680, L137

Romano, P., Sidoli, L., Cusumano, G., et al. 2009, ApJ, 696, 2068

Romano, P., Krimm, H. A., Palmer, D. M., et al. 2014, A\&A, 562, A2

Sguera, V., Barlow, E. J., Bird, A. J., et al. 2005, ATel, 444, 221

Sguera, V., Bird, A. J., Dean, A. J., et al. 2006, ATel, 873, 1

Sidoli, L., Paizis, A., \& Mereghetti, S. 2006, A\&A, 450, L9

Sidoli, L., Romano, P., Mereghetti, S., et al. 2007, A\&A, 476, 1307

Sidoli, L., Romano, P., Mangano, V., et al. 2008, ApJ, 687, 1230

Smith, D. M., Main, D., Marshall, F., et al. 1998, ApJ, 501, L181

Smith, D. M., Heindl, W. A., Swank, J. H., Harrison, T. E., \& Negueruela, I. 2003, ATel, 182, 1

Smith, D. M., Negueruela, I., Heindl, W. A., Markwardt, C. B., \& Swank, J. H. 2004, BAAS, 36, 954

Smith, D. M., Heindl, W. A., Markwardt, C. B., et al. 2006, ApJ, 638, 974

Sunyaev, R., Lutovinov, A., Molkov, S., \& Deluit, S. 2003, ATel, 181, 1

Torrejón, J. M., Negueruela, I., Smith, D. M., \& Harrison, T. E. 2010, A\&A, 510, A61

Treves, A., Maraschi, L., \& Abramowicz, M. 1988, PASP, 100, 427

Turler, M., Shaw, S. E., Kuulkers, E., et al. 2006, ATel, 790, 1

Ubertini, P., Lebrun, F., Di Cocco, G., et al. 2003, A\&A, 411, L131

van den Heuvel, E. P. J. 1976, in Structure and Evolution of Close Binary Systems, eds. P. Eggleton, S. Mitton, \& J. Whelan, IAU Symp., 73, 35

van den Heuvel, E. 2012, in Proc. of An INTEGRAL view of the high-energy sky (the first 10 years) - 9th INTEGRAL Workshop and celebration of the 10th anniversary of the launch (INTEGRAL 2012), 15-19 October, Bibliothèque Nationale de France, Paris, France

Voss, R., \& Ajello, M. 2010, ApJ, 721, 1843

Walter, R., \& Zurita Heras, J. 2007, A\&A, 476, 335

Winkler, C., Courvoisier, T. J.-L., Di Cocco, G., et al. 2003, A\&A, 411, L1 